\title{
El papel del Estado en los procesos de urbanización en el municipio de Itagüí, Colombia ${ }^{1}$
}

\section{Juliana Ríos Medina}

Estudiante de trabajo social, integrante del semillero de investigación Territorio de la Universidad Pontificia Bolivariana. Correo electrónico:

Aprobado: 4 de junio de 2020 Recibido: 3 de diciembre de 2019 DOI: http://dx.doi.org/10.18566/rfts.v35n35.a01 julianarios0796@gmail.com

1 El presente artículo surge como resultado del trabajo de grado dirigido por la docente Luz Stella Carmona Londoño. 


\section{Resumen}

En la cuenca Los Gómez-El Ajizal, (veredas del municipio de Itagüí), se han presentado procesos de expansión y urbanización en los últimos diez años. Esto puede responder a que los territorios rurales en el sistema capitalista se han convertido en la posibilidad de acumulación de los intereses económicos a partir de la transformación de los usos del suelo, sin embargo, el capital requiere de un agente que respalde su proceso de reproducción, este es el Estado. A partir de la revisión de los planes de desarrollo y el POT del municipio de Itagüí, se indagó sobre las decisiones que llevaron a dichos procesos.

Palabras clave

Urbanización, Territorio, Estado, Itagüí.

\section{Abstract}

In the Los Gómez-El Ajizal basin, (paths of the municipality of Itaguií; expansion and urbanization processes have been presented in the last 10 years. This may answer that the rural territories in the capitalist system have become the possibility of accumulating economic interests from the transformation of land uses, however, Capital requires an agent to support its reproduction process, this is the State. Based on the review of the development plans and the POT, the decisions that led to these processes were investigated.

Key words

Urbanization, Territory, State, Itagüí 


\section{Introducción}

El semillero de investigación Territorio, de la Universidad Pontificia Bolivariana de Colombia, conformado principalmente por estudiantes de Trabajo Social, se ha preocupado entender la realidad social, particularmente de las áreas rurales en contextos metropolitanos. Es por esta razón que se desarrolló una investigación que indagó por las modificaciones del uso del suelo y el paso de lo rural a lo urbano con intereses específicos. La pregunta que sirvió como orientación para la investigación fue: ¿Cómo han sido las transformaciones territoriales de la cuenca Los Gómez, Itagüí a partir de la relación sociedad-Estado-privado en el proceso de urbanización en el periodo $2000-2017$ ?

El municipio de Itagüí es uno de los diez entes que conforman el Área Metropolitana del Valle de Aburrá, y está ubicado en la parte centro del departamento (Alcaldía de Itagüí, 2019). Itagüí se encuentra sobre la orilla izquierda del río Medellín. Los municipios limítrofes son: al oriente Envigado y Sabaneta; al suroccidente La Estrella, al norte Medellín y al occidente el corregimiento de San Antonio de Prado (Mesa ambiental del municipio de Itagüí, 2019); ver figura 1. 


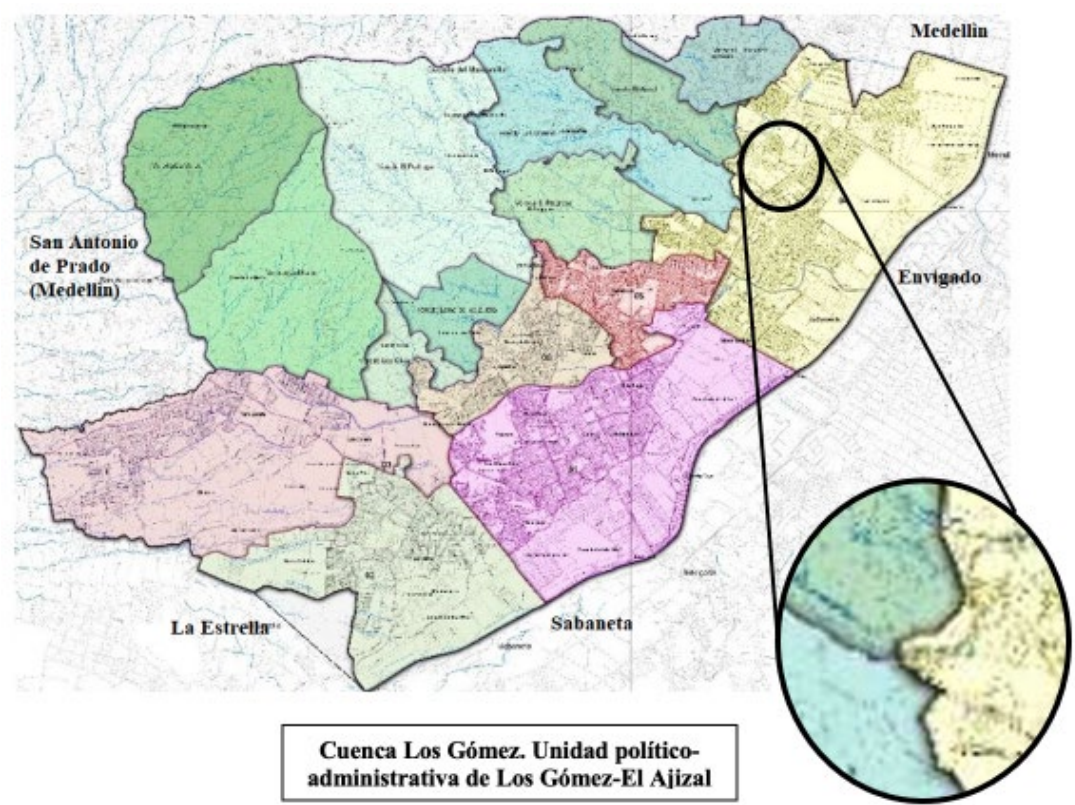

Fuente: imagen modificada a partir de Calderón y Goez (2019).

Con respecto a lo físico-espacial, es el tercer municipio más pequeño del país con una extensión de $21,09 \mathrm{~km}^{2}: 12,17 \mathrm{~km}^{2}$ en la zona urbana y $8,91 \mathrm{~km}^{2}$ en la zona rural. Las alturas están entre los 1.590 y los 2.180 metros sobre el nivel del mar. También es el municipio más densamente poblado y uno de los más industrializados del país. Gran parte del territorio está destinado al uso urbano. El nuevo suelo de expansión urbana comprende 130 hectáreas (Mesa Ambiental del Municipio de Itagüí, 2019). El territorio está constituido por elementos construidos y naturales. Entre los últimos se reconoce el río Medellín y las quebradas. Al hablar de elementos hidrográficos importantes en el municipio, según Morales Soto (1979), está la quebrada Doña María, la cual es uno de los principales afluentes del río Medellín. Las zonas cercanas de la quebrada Doña María, de una u otra forma, se han convertido en un importante sector de crecimiento industrial. Morales Soto (1979) menciona 
que por la disposición de mano de obra y por sus condiciones climáticas, se ha convertido en el sector industrial más importante del Valle de Aburrá. La quebrada Doña María ha tenido un papel trascendental en la industrialización del municipio de Itagüí, ya que ha sido desde hace tiempo, la principal fuente de agua para las industrias de la zona. Actualmente continúa siendo un referente industrial que cada día crece más, con respecto a la industria y a su población.

Entre los elementos construidos se encuentra el Metro. Este se ha convertido en un referente estructurante de Itagüí pues permite el acceso al municipio desde cinco estaciones, como se ve en la figura 2. También es un impulsor de la urbanización en el Valle de Aburrá. Se identifica como una mega infraestructura, su carácter de transporte masivo y los cambios que generó su construcción en los puntos por donde cruza han sido estructurales, sociales y económicos.

\section{Figura 2. Estaciones del Metro ubicadas en Itagüí}

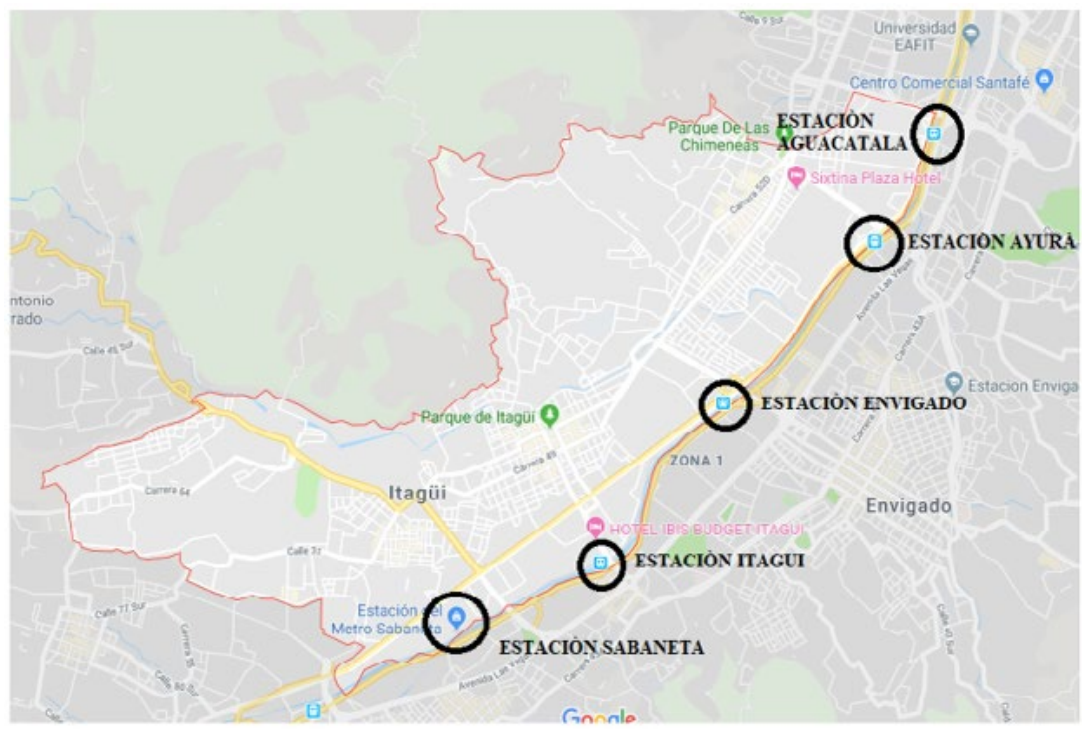

Fuente: Imagen con modificaciones a partir de Google (2019) 
La zona rural del municipio de Itagüí está dividida en un corregimiento y diez veredas, esto de acuerdo con el artículo 1 de la Ley 136 de 1994², que se agrupan en cuatro sectores. El nombre del corregimiento es el Manzanillo, allí se localiza la cuenca de la quebrada Los Gómez, al noroccidente del municipio. La cuenca está comprendida por dos unidades políticoadministrativas, la vereda Los Gómez y El Ajizal.

En la siguiente figura se puede observar el crecimiento de la población de Itagüí en la zona urbana en el periodo de 1964 al 2015. Entre el 2005 y el 2015 hubo un crecimiento de población de $26.697^{3}$ personas (ver figura 3 ), lo que llevó a nuevas demandas de vivienda. En la siguiente figura también se puede observar cómo el crecimiento de la población ha sido exponencial desde 1964.

Figura 3. Crecimiento de la población de Itagüí en la zona urbana

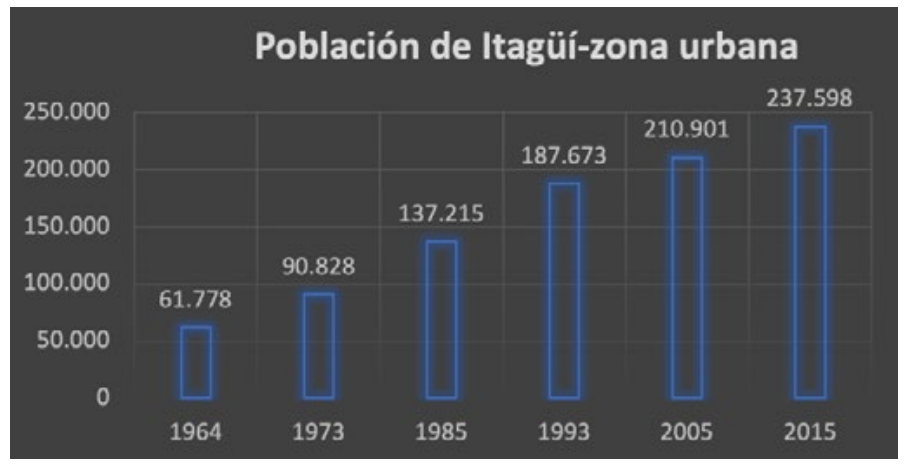

Fuente: elaboración propia a partir de DANE (2018).

2 Ley 136 de 1994. Capítulo 1. Artículo 1. Definición. El municipio es la entidad territorial fundamental de la división político-administrativa del Estado, con autonomía política, fiscal y administrativa dentro de los límites que le señalen la Constitución y la ley y cuya finalidad es el bienestar general y el mejoramiento de la calidad de vida de la población en su respectivo territorio.

Capítulo VII. Artículo 117. Comunas y corregimientos. Con el fin de mejorar la prestación de los servicios y asegurar la participación de la ciudadanía en el manejo de los asuntos públicos de carácter local, los concejos podrán dividir sus municipios en comunas cuando se trate de áreas urbanas y en corregimientos en el caso de las zonas rurales.

3 En un aproximado de 3 personas por hogar -según el DANE (2018) como tamaño promedio de los hogares en Colombia- serían necesarias 8.899 viviendas nuevas que debieron construirse en el municipio. 
La cuenca Los Gómez se caracteriza históricamente por ser una zona rural con actividades agrícolas y la elaboración de ladrillos. Sin embargo, por su cercanía con la cabecera municipal en los últimos diez años ha tenido un proceso de expansión de actividades urbanas, principalmente con la densificación y construcción de urbanizaciones cerradas. Esto ha generado transformaciones en el territorio, físicas, sociales y en las formas de vida. Estos cambios no son exclusivos de la zona rural de Itagüí, es una particularidad de las zonas rurales dentro de los contextos metropolitanos, en este caso el Área Metropolitana del Valle de Aburrá -AMVA-.

El AMVA está ubicada en el centro-sur del departamento de Antioquia, Colombia, en un valle interandino de la cordillera Central. La corriente del río Medellín cruza el valle de sur a norte, nace en el municipio de Caldas y confluye con el río Grande a la altura de Puente Gabino, entre los municipios de Don Matías y Yolombó. El valle cuenta con unos $60 \mathrm{~km}$ de longitud y una amplitud promedio de $30 \mathrm{~km}$, mientras que en su parte más ancha mide entre 80 y $90 \mathrm{~km}$.

La población del Valle de Aburrá está distribuida en diez municipios: Caldas, La Estrella, Sabaneta, Itagüí, Envigado, Medellín, Bello, Copacabana, Girardota y Barbosa (ver figura 4). El AMVA 4 tiene una extensión de 1.152 $\mathrm{km}^{2}$, el $94 \%$ corresponde a los $1.251,08 \mathrm{~km}^{2}$ de extensión de la cuenca del río Aburrá-Medellín. De los $1.152 \mathrm{~km}^{2}, 340 \mathrm{~km}^{2}$ son suelo urbano y $812 \mathrm{~km}^{2}$ son suelo rural (Área Metropolitana, 2007). La población del Valle de Aburrá ascendía a 3.312.165 habitantes en 2005 según el censo nacional realizado por el DANE; no obstante, en el censo de 2018 la cifra fue de 3.726.219 habitantes (DANE, 2019), los cuales se asientan principalmente en el suelo urbano.

4 Ley 1625 de 2013. Artículo 2. Las áreas metropolitanas son entidades administrativas de derecho público, formadas por un conjunto de dos o más municipios integrados alrededor de un municipio núcleo, vinculados entre sí por dinámicas e interrelaciones territoriales, ambientales, económicas, sociales, demográficas, culturales y tecnológicas que para la programación y coordinación de su desarrollo sustentable, desarrollo humano, ordenamiento territorial y racional prestación de servicios públicos, requieren una administración coordinada. 


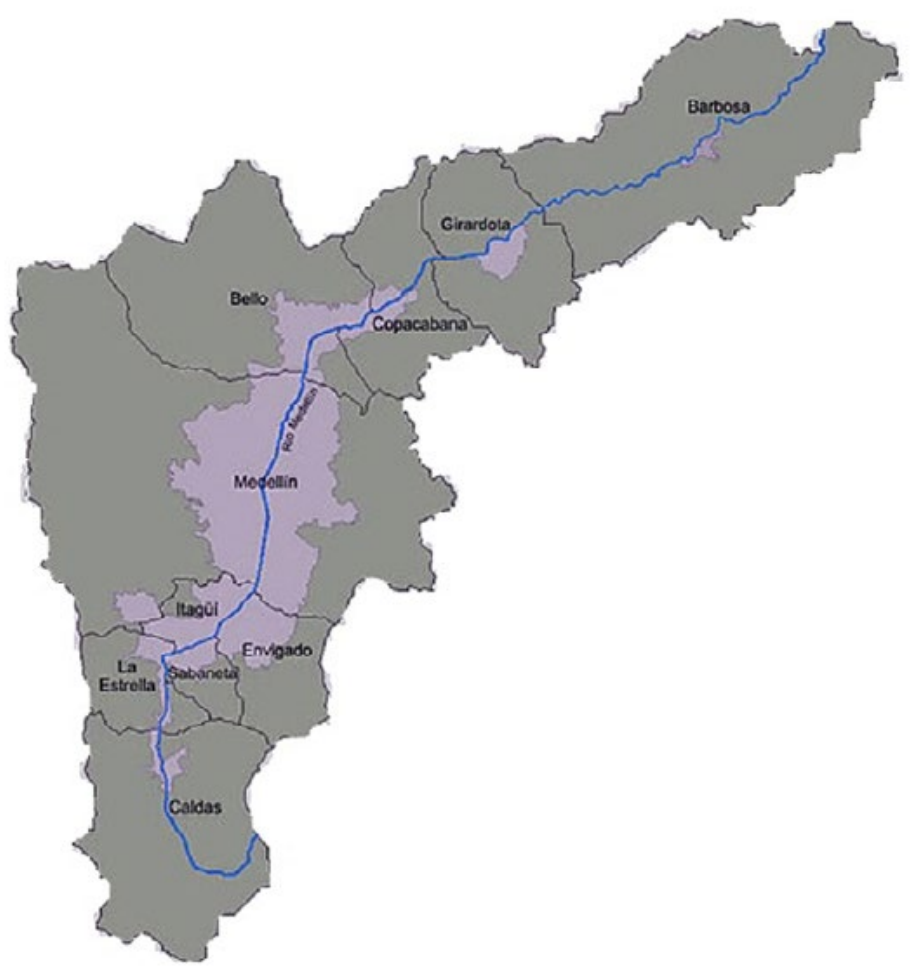

Fuente: imagen tomada de Unimedios (Agencia de noticias de UN) (2010).

Unas de las condiciones geográficas más destacable del valle es que está enmarcado por una topografía irregular y pendiente, que oscila entre 1.300 y 2.800 metros sobre el nivel del mar. Las cordilleras que lo encierran dan lugar a la formación de diversos microclimas, saltos de agua y bosques. E1 suelo posiblemente urbanizable es reducido. Por lo anterior, se empiezan a presentar tres fenómenos: el primero es la presión hacia otras regiones aledañas por fuera del valle, por ejemplo, del oriente antioqueño con el túnel de Oriente. El segundo, es la densificación del valle donde no existe la posibilidad de expansión, lo que hace necesario crecer en altura, y por último 
la presión por urbanizar el suelo rural, que por sus condiciones geográficas es urbanizable y el precio es menor (suburbanización) ${ }^{5}$.

En Colombia ${ }^{6}$, el suelo rural próximo a las ciudades, ofrece un panorama crítico por su situación de frontera entre un campo que ha sido abandonado como proyecto de desarrollo económico y social en los últimos sesenta años, y una ciudad sometida a un proceso de urbanización intensivo. Además, en este período se presentó un cambio en la demografía, la economía y la cultura del país. Este escenario rural presenta las siguientes características: urbanización incontrolada y espontánea de vivienda VIS 7 , viviendas de recreo, periurbanización precaria siguiendo el patrón de hábitat urbano; comunidades rurales empobrecidas, desarraigadas y trabajos de baja calificación; deterioro progresivo de los ecosistemas, la biodiversidad, las fuentes de agua y el paisaje agrario; ruralidad homogeneizada y, finalmente, una política pública rural fragmentada y de bajo impacto (Arango-Escobar, 2008).

A esta situación se le debe sumar que la apertura económica que se generó, no tuvo en cuenta las condiciones particulares del país, la economía se desestabilizó, las actividades tradicionales (agrícolas, ganaderas, silvícolas) del campo casi desaparecieron, se aumentó el desempleo, y de manera alarmante se incrementó la crisis de la sociedad colombiana (Londoño, 1998). Además, si se agrega la dinámica poblacional de Colombia, el panorama es crítico. En la siguiente figura se puede observar el crecimiento de la población de Colombia en la zona urbana en el periodo de 1973 al 2018. Entre el 2005 y

5 Suelo suburbano. Son las áreas ubicadas dentro del suelo rural, en las que se mezclan los usos del suelo y las formas de vida del campo y la ciudad, puede ser objeto de desarrollo con restricciones de uso, de intensidad y de densidad, garantizando el auto abastecimiento en servicios públicos domiciliarios (Alcaldía de Itagüi, 2000).

6 Su territorio es de $1.141 .748 \mathrm{~km}^{2}$ a los cuales se suman la plataforma marina y submarina. En Colombia hay treinta y dos departamentos (dirigidos por gobernadores); 1.123 municipios (dirigidos por alcaldes); cinco entidades territoriales con una administración especial (Bogotá y las ciudades puerto de Cartagena, Barranquilla, Santa Marta y Buenaventura); las entidades territoriales indígenas; y los territorios colectivos adjudicados a comunidades afrocolombianas en zonas del Pacífico. El Estado se divide en las tres ramas del poder público: la legislativa, la ejecutiva y la judicial (PNUD, 2019, párr.13).

7 Vivienda de interés social (VIS): es aquella que reúne los elementos que aseguran su habitabilidad, estándares de calidad en diseño urbanístico, arquitectónico y de construcción cuyo valor máximo es de ciento treinta y cinco salarios mínimos legales mensuales vigentes (135 SMLM) (Minvivienda, 2009, párr.16). 
el 2018 hubo un crecimiento de población de 5.696 .919 personas (ver figura 5), también se puede constatar que el crecimiento de la población ha sido exponencial desde 1973.

Figura 5. Crecimiento de la población de Colombia en zona urbana

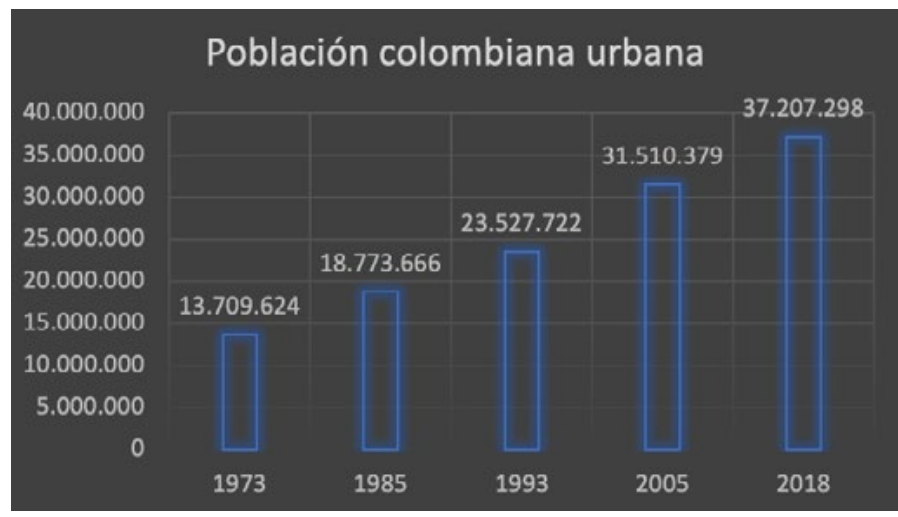

Fuente: elaboración propia a partir de DANE (2018).

Debido a los fenómenos descritos, a la dinámica poblacional y la necesidad de planificar el territorio, Colombia a finales de la década del 90 expidió la Ley 388 de 1997 que estableció lineamientos para que el territorio fuera ordenado por medio de planes territoriales. La finalidad de esta ley es que los municipios por medio de su autonomía ordenen el territorio, para permitir el uso equitativo y racional del suelo, preservando el patrimonio cultural y ecológico. Además, para prevenir desastres donde existe riesgo. Esta ley debe garantizar la utilización del suelo que tienen los dueños de las propiedades para hacer efectivos los derechos constitucionales a la vivienda y a los servicios públicos, velar por el espacio público y su creación. Así mismo, en el artículo 9 de la misma ley aparece la figura del Plan de Ordenamiento Territorial (POT), que es el instrumento básico para desarrollar la organización del territorio municipal. El POT es el conjunto de normas adoptadas para orientar y administrar el desarrollo físico del territorio y la utilización del suelo. De esta manera, se convierte en uno de los instrumentos del Estado en este caso a escala local, en cabeza de 
las alcaldías que define los usos, ocupación del suelo e intensidad de uso, aparentemente respondiendo a necesidades sociales y económicas que favorezcan a la población.

Aunque el tema del ordenamiento del territorio es relativamente nuevo para Colombia, es el resultado de un proceso histórico mundial. A partir del surgimiento del capitalismo, la necesidad de controlar la tierra y delimitarla se hace cada vez más importante. El capitalismo como forma de producción encuentra terreno firme:

Para su formación a nivel político a través de la alianza establecida entre el capital comercial y la realeza, y a nivel ideológico, a través de doctrinas mercantilistas. La alianza de la burguesía comercial con el rey permitió la formación de los Estados Nacionales Absolutos. El orden capitalista se impuso al orden feudal convirtiendo la tierra en mercancía; al mismo tiempo, se impusieron nuevas leyes sobre los intereses de monopolio de las corporaciones, al finalizarse dichas leyes, el capitalismo se tomó la ciudad y transformó el carácter de la producción artesanal urbana. La formación de los Estados nacionales absolutistas permitió la densificación de la población en la medida en que el aparato político-administrativo que apoyaba al Estado condujo al surgimiento de una gran burocracia y la formación de ejércitos permanentes (Sposito, 1988, p. 41).

Todo lo anterior está influenciado por el papel que el Estado juega, para Mészáros (2001), en su libro Más allá del capital, la configuración del Estado moderno es un requisito absoluto para asegurar y cuidar permanentemente los logros productivos del sistema. La llegada del capital al dominio en la producción material y el avance de las prácticas políticas totalizadoras en forma del Estado moderno van de la mano. El Estado debe ajustar continuamente sus funciones reguladoras para ponerlas en sintonía con el proceso dinámico cambiante de reproducción socioeconómica, complementando y reforzando políticamente la dominación del capital contra las fuerzas que se atrevan a retar las "flagrantes iniquidades de la distribución y el consumo” (Mészáros, 2001, p. 75). 
Lo anterior implicaría que el Estado desde sus acciones, decisiones, y la creación de leyes y ajustes, quiere responder a la función por la cual se le dio génesis: cuidar del capital. Como lo ratifica Mészáros (2011) citado por Ribeiro (2014): la función del Estado es cuidar los intereses particulares para que la rueda del capitalismo continúe (p.128, 185).

Si se entiende que el Estado ejerce un poder que se materializa en los territorios nación, es importante citar la concepción que los geógrafos han dado al Estado: "El Estado existe cuando una población ubicada en un territorio ejerce su propia soberanía” (Raffestin, 1980, p. 21). A partir de lo anterior, para este autor, se movilizan tres signos para caracterizar al Estado: la población, el territorio y la autoridad. Toda la geografía del Estado deriva de esta tríada, en la cual se crea una relación que puede expresarse, como el papel que el espacio y el tiempo juegan, así:

Los habitantes de un valle fértil viven en abundancia de productos naturales; una fuente riega sus tierras (...). Alguien agarra la fuente, vende agua a usuarios; redoblan sus esfuerzos para pagar la tarifa del propietario de la fuente, se convierte en señor del valle: rico y poderoso (Raffestin, 1980, p. 29).

Para el autor, en esta relación hay "abuso" de un polo de la relación sobre el otro. Raffestin (1980) plantea que el poder en el territorio no solo puede ser visto por el que ejerce el Estado, sino que en el territorio se generan variadas formas y relaciones de poder, evidenciadas en interacciones sociales, económicas y culturales; en estas se encuentra energía y acciones determinadas como también simbólicas (pp.112-113).

Por otro lado, Raffestin (1980), menciona que el territorio es un activo particular, barrera y recurso, contenido y continente, todo al mismo tiempo (p. 45). El territorio es el espacio político por excelencia, es el campo de acción. Y agrega que el Estado puede pretender lograr una distribución balanceada de la población en el territorio y por medidas tentativas, y arreglos e inversiones generadoras de empleo; por ejemplo, puede remodelar el mapa de distribución de la población. Pero sin duda, hay empresas que tienen un interés principal donde invierten, allí juegan el papel primordial sobre la distribución de la población. Las empresas buscan lograr la mayor movilidad geográfica posible para cumplir con sus necesidades. 
Ahora bien, si se considera que el proceso de mayor transformación en los territorios es la urbanización, como se evidenció en los párrafos anteriores, cuando se expuso el contexto de Itagüí y de Colombia, es importante definir qué se entenderá por este proceso en este artículo. Según Sposito (1988), la urbanización es un proceso que surge por el capitalismo y la industrialización. Ella menciona que el desarrollo de la urbanización no es solo un requisito para el desarrollo industrial, sino que también ha cambiado el carácter de la ciudad al darle determinantemente un rasgo productivo y convertirla en el "centro" de control y gestión de la economía capitalista, relegando la producción agrícola (p. 4). La autora da indicios del surgimiento de la urbanización en los países "periféricos" y la menciona como una de las formas producto del desarrollo del capitalismo, que se traduce en la articulación de las relaciones económicas, sociales y políticas existentes entre los países "desarrollados" y "subdesarrollados"(pp.73, 84). En otras palabras, se puede decir que la urbanización es el resultado del proceso de transnacionalización de la industria occidental (el "centro"), que llega a las periferias y desorganiza, cambia y se apropia de las formas tradicionales de producción de estos países (Sposito, 1988, pp.5, 74, 75 ).

\section{Problema}

Itagüí es un municipio que en su proceso histórico ha llegado a ser uno de los más densos del país y el más industrializado del Valle de Aburrá; las dos condiciones han llevado a nuevas necesidades poblacionales, sociales, económicas, habitacionales, entre otras. Estas situaciones no pueden ser ajenas a los entes que administran el territorio y que empiezan a buscar alternativas. Con los aumentos de población es imprescindible expandir la ciudad, y es aquí donde aparecen las zonas rurales y terrenos de usos de protección agrícolas o mineros que "estorban”, y no permiten crecer la ciudad aceleradamente. Entre las veredas de El Ajizal y Los Gómez se empiezan a ver las decisiones administrativas que favorecen el cambio de usos de suelo y permiten la expansión de lo urbano hacia lo rural.

Esta transformación ocurrida en un tiempo corto (diez años) ha creado un conflicto entre los modos de vida rurales (prácticas agropecuarias, acueductos comunitarios, entre otros) y las dinámicas urbanas por la disputa 
del territorio. Este fenómeno de urbanización no es exclusivo de Itagüí ni de estas veredas, por el contrario, ocurre en toda el área metropolitana aumentando las tensiones ya que el Valle de Aburrá tiene poco suelo urbanizable y sus condiciones topográficas no le permiten más expansión, a excepción de hacerlo verticalmente. Es necesario señalar que el proceso de urbanización es un fenómeno mundial que se ha trasladado, con el tiempo, desde los países "del primer mundo" hasta los países "subdesarrollados". No es un proceso nuevo si se tiene en cuenta que es consecuencia del capitalismo y la industrialización.

La acumulación de capital requiere de mano de obra barata y calificada con fácil acceso. También es necesaria la actualización en las formas de producción, es por ello que se vuelve fundamental "presionar" para que las ciudades se conviertan en el centro de las actividades humanas, donde se encuentra la oferta más amplia del mercado y de formas de vida, y van apareciendo demandas más fuertes por parte de la población. Las presiones que estas dinámicas generan en el campo son grandes, como la necesidad de una mayor producción para satisfacer a la ciudad, pero ofreciendo mínimas garantías de permanencia.

Por otro lado, existe la necesidad de ampliar esa ciudad y el recurso para esto es el suelo rural. Si en las zonas rurales no se tienen garantías de permanencia, acceso a servicios públicos y subsistencia, las personas que habitan estos territorios deben buscar otros espacios para "sobrevivir", obligando a muchos a trasladarse a las ciudades y cambiar las relaciones que se tejen en los territorios, en la medida que aumente la población de la ciudad, es necesaria la construcción de más viviendas, lo que lleva a la densificación y en muchas ocasiones a crisis para abastecer de servicios públicos y garantías de calidad de vida a las personas.

Se observa que el proceso de urbanización puede ir por encima de las condiciones naturales del territorio, como se resalta en Itagüí. En muchas ocasiones presionando para la expansión urbana, pero sin un suelo acorde para ello, irrumpiendo en el suelo rural para cumplir con la demanda exigida. Sin embargo, este proceso no se da al azar, por el contrario, detrás de estas demandas, dinámicas y presiones existen intereses que ocasionan que esto ocurra, en ocasiones, a ritmos acelerados. 
Es aquí donde el Estado cumple un papel fundamental, el cual es velar por el bienestar de las personas que habitan el territorio, por medio de planes, programas y proyectos, planes de desarrollo y el plan de ordenamiento territorial (POT) con el fin de garantizar los derechos de los ciudadanos. Sin embargo, en el caso de Itagüí, el POT va dando condiciones para el proceso de urbanización de lo rural al decretar, en su artículo 31 del Acuerdo 020 de 2007, a las veredas Los Gómez y El Ajizal (pertenecientes a la cuenca de los Gómez) como suelo de expansión urbana, cambiando el uso tradicional de la fabricación de ladrillos en suelo para urbanizar, generó así diversos cambios en las formas de vida de los habitantes de este territorio.

Es por lo anterior, que vale la pena reflexionar sobre el papel del Estado y sus formas de actuar, si corresponden y se alinean con lo que predica la ley.

\section{Pregunta}

De acuerdo con lo expuesto anteriormente se pretende por medio de este artículo responder la siguiente pregunta: ¿Cuál es el papel del Estado en el proceso de urbanización en el municipio de Itagüí en la parte baja de la cuenca Los Gómez en los últimos 20 años?

\section{Materiales y métodos}

Fue necesaria la revisión de las páginas oficiales del DANE, de la Alcaldía de Itagüí y del Área Metropolitana para la obtención de información: censos, planes de desarrollo y planes de ordenamiento territorial. Con lo anterior se creó una matriz para su análisis y contraste entre ellos, haciendo un rastreo a partir de categorías. 


\section{Discusión de resultados}

Itagüí y el área metropolitana son similares en situación de una realidad que se vive en el contexto nacional, como es el crecimiento de la urbanización. Para describir los intereses del Estado en este caso y en lo local, los instrumentos más importantes son los planes de desarrollo y el POT (Plan de Ordenamiento Territorial). Para entender las apuestas de los planes de desarrollo en cuanto al proceso de transformación en el área de estudio, parte baja de la vereda El Ajizal y Los Gómez, fueron analizados los últimos cuatro, es decir, los comprendidos en los siguientes periodos: 2004-2007; 2008-2011; 2012-2015 у 2016-2019.

Analizando los planes de desarrollo de los diferentes periodos, se percibe la disparidad con respecto a la profundización de los diversos temas que son relevantes, se observa cómo en algunos planes se centran más en unos temas y como, por el contrario, se descuidan otros, algo que está presente en todos los planes.

En cuanto a la visión, los planes de desarrollo tienen un eje estructurante: lo económico. Para el plan del 2004-2007, la visión es descentralizar el poder y fomentar el desarrollo social, económico y político, poniendo estos tres pilares como el eje de su accionar.

El periodo 2008-2011 también se centra en lo económico, esperando para el año 2011 alcanzar "más desarrollo para todos", un nivel de calidad para los habitantes y crecimiento productivo. Algo semejante ocurre para el periodo 2012-2015, pues enfoca su visión en lo económico, queriendo convertir a Itagüí en una ciudad competitiva, productiva, con un valor de innovación, con asociativa empresarial, empleos y alianzas público-privadas. Hay que señalar que en el plan de 2016-2019 se pretende que para el 2025 Itagüí sea un municipio territorial y socialmente equilibrado, con cierre de brechas, además de comercial, industrial y de servicios. Si la visión sirve para las proyecciones del municipio, estas muestran el camino a tomar con un enfoque económico, que desde las diversas administraciones pretenden impulsar; desde aquí las decisiones, proyectos y programas deberán ir acorde al cumplimiento de este objetivo. 
En relación al territorio, algunos planes abordan las directrices del Plan de Ordenamiento, como el plan de 2004-2007 y el de 2012-2015, y otros lo nombran de manera ambigua, como el plan de 2008-2011, que habla de la consolidación del territorio, pero no aclara el cómo. Finalmente, el plan de 2016-2019 busca la aplicación de instrumentos de gestión para el desarrollo territorial y ambiental, además de complementar la planificación económica y social con la dimensión territorial, racionalizar las intervenciones sobre el territorio y orientar su desarrollo y aprovechamiento sostenible. De nuevo nos encontramos con un asunto que inicia desde lo económico.

Con respecto al uso del suelo ${ }^{8}$, el plan de 2004-2007, pretende la integración de este en función de la industria y el comercio, se reconoce de nuevo la importancia de fortalecer los mecanismos que utiliza el capital para mantener el control. También habla de liberar áreas para el espacio público y busca contar con la formulación de planes parciales para zonas de expansión urbana. Asimismo, se enfoca más en la expansión de las zonas urbanas, procurando equipamiento y mejoras para garantizar los usos de industria y comercio. Lo anterior deja expuestas las intenciones administrativas de preparar los territorios para la expansión del suelo urbano.

En lo que se refiere al periodo de 2008-2011, el objetivo es el suelo, para cubrir necesidades habitacionales y de mejoramiento integral, enfocándose en los desarrollos de vivienda de interés social. Mientras, en el plan de 2012-2015, lo relacionado con el uso del suelo se centra en lo dictado por el POT, y tiene como propósito convertir al municipio en una ciudad metrópoli. Por otro lado, se procuró realizar la estratificación de centros poblados y rurales, para reflejar la realidad de las condiciones de los predios y ajustar los estratos socioeconómicos, los cuales pretenden ser cambiados en ciertas zonas, aumentando por consiguiente los impuestos y los precios del suelo.

8 En el Decreto 1203 de 2017, artículo 2.2.6.1.3.1. y numeral 2, respecto al suelo se dice: "es el dictamen escrito por medio del cual, el curador urbano o la autoridad municipal o distrital competente para expedir licencias o la oficina de planeación o la que haga sus veces, informa al interesado sobre el uso o usos permitidos en un predio o edificación, de conformidad con las normas urbanísticas del Plan de Ordenamiento Territorial y los instrumentos que lo desarrollen. La expedición de estos conceptos no otorga derechos ni obligaciones a su peticionario y no modifica los derechos conferidos mediante licencias que estén vigentes o que hayan sido ejecutadas. 
Concerniente al periodo de 2016-2019, en este plan se presenta una descripción del municipio a partir de la contradicción de tener una zona urbana densa, al lado de una pequeña zona rural olvidada; posteriormente continúa comentando sobre los desequilibrios del territorio, siendo este poco sostenible y sustentable, y sin una línea ambiental clara. Todo esto enfocado en la importancia de organizar el territorio "para hacer sostenibles y compatibles diversas vocaciones del suelo, usos y aprovechamientos" (Concejo Municipal de Itagüí, 2016, p.10), y la utilización de actos administrativos que reglamentan los usos del suelo para establecimientos abiertos al público. Lo anterior, denota un interés de las diversas administraciones por el equipamiento de zonas públicas con miras a la expansión de suelo urbano, continuando con la relegación del campo, además, del interés latente de ajustar la estratificación, con miras a los cambios del uso del suelo, aumento de impuestos y servicios públicos.

En consideración al tema de la vivienda, en el plan de 2004-2007, se mencionan varias acciones como: la promoción de viviendas de interés social en suelo de expansión, el mejoramiento de entornos en zonas de rehabilitación, el desarrollo de proyectos de vivienda en las zonas de redensificación contempladas en el POT, los estímulos a programas para el desarrollo de proyectos de vivienda en el suelo de expansión urbana, y el impulso de programas de renovación urbana. También define las metas a conseguir, que son: construcción de 300 VIS nuevas en zona urbana de Itagüí, mejoramiento de entornos de 200 viviendas en zonas de rehabilitación determinadas en el POT y compra de dos lotes en zonas de expansión urbana.

Por otro lado, algunas de las oportunidades que el plan contempla son: el interés en adelantar programas públicos o privados que contribuyan a desarrollar soluciones de vivienda de interés social, la localización geográfica sobre el eje del río y como "puerta del suroeste", la tradición y dinámica industrial y el desarrollo comercial actual y el acceso a proyectos de carácter metropolitano. Además, el plan tiene en cuenta ciertas debilidades que se tienen frente al tema de vivienda, como es la continuidad de un alto déficit de vivienda y la especulación y monopolio de los pocos terrenos de expansión. Del mismo modo, propende por el mejoramiento urbanístico del municipio generando programas de vivienda en suelos de expansión. Es clara la apuesta por desarrollar los suelos de expansión, con estos, antes suelos rurales, intenta cubrir la demanda de viviendas que se da aceleradamente en 
el municipio. Según los datos del POT, la extensión de lo rural es de 42,29 hectáreas y así se ha mantenido desde el 2010 al 2014, mientras que para el 2007, en las memorias justificadas del POT se manifiesta que el suelo urbano es de $12,17 \mathrm{~km}^{2}$, cuando la realidad es que este aumenta cada vez más. Tal situación evidencia y genera una contradicción, ya que no puede seguir existiendo el mismo suelo rural, cuando el suelo urbano ha crecido y su única forma de hacerlo es hacia lo rural.

En cuanto al periodo de 2008-2011, tiene como meta fomentar la calidad y productividad de los proyectos habitacionales, por medio del aprovechamiento racional del suelo. Este plan centra su accionar en estimular la oferta de nuevas viviendas VIS.

Alineado al anterior, el plan de 2012-2015 tiene como objetivo de vivienda garantizar a las familias de Itagüí el acceso digno. Asimismo, cuenta con unos indicadores que pretenden conocer el área aprobada para construcción de vivienda en hectáreas, el suelo habilitado urbanizado para vivienda de interés social en hectáreas por año, y el déficit cuantitativo de vivienda.

Por su parte, en el periodo 2016-2019 se habla de generar todos los actos administrativos y contratos relativos a la gestión de la política sectorial de vivienda en el municipio de Itagüí, en la que se incluye la compra y transferencia de bienes muebles e inmuebles. También propone trabajar por el mejoramiento de los entornos barriales y veredales, la construcción de viviendas, promover el desarrollo de proyectos en la centralidad sur del municipio y el desarrollo integral del corregimiento El Manzanillo.

Con respecto a la movilidad se destaca la construcción de la conexión Pedregal-Los Gómez, la conexión El Ajizal-El Porvenir y la conexión La Loma de los Zuleta-El Progreso. Estas labores se orientan al mejoramiento de las vías de acceso dentro del municipio, de la parte urbana con las zonas rurales, en puntos cercanos a la expansión del suelo urbano. Básicamente, lo que se procura con estas obras es un equipamiento del suelo con cambios a partir de transformaciones significativas y planeadas para que este, próximamente, pueda ser la puerta a la urbanización que permita la transición de lo rural a lo urbano. 
Todo lo anterior descrito y mencionado en este artículo, tiene como finalidad acercarse al papel que cumple el Estado en los procesos de urbanización, en este caso específico en la cuenca Los Gómez-El Ajizal, para mostrar, desde una perspectiva crítica, cómo las administraciones municipales a lo largo de sus trayectorias, van fijando los cambios en el suelo municipal y esto desemboca en cambios, no solo estructurales, sino en dinámicas sociales y económicas que afectan a las poblaciones que habitan los territorios.

Si se agudizan los sentidos, se puede observar con claridad a lo que se refería Mészáros (2001), cuando decía que el Estado debe ajustar continuamente sus funciones reguladoras para ponerlas en sintonía con el proceso socioeconómico dinámico de reproducción, complementando y reforzando políticamente la dominación del capital; algo que se ve reflejado en cada plan de desarrollo revisado desde el 2004 hasta el 2019 en los que la función ha sido poner la maquinaria del Estado, las leyes y normas al servicio del capital.

Si se presta atención a los altos índices de aumento de población en el municipio, es fundamental pensar hacia dónde debe crecer la ciudad para satisfacer las necesidades de vivienda. Las ciudades como centros del desarrollo industrial y comercial requieren de una gran cantidad de viviendas para contener las personas que desean vivir en estos centros dotados de bienes y servicios a su disposición, pero ¿Qué sucede cuando el número de personas crece aceleradamente y las condiciones geográficas no son las adecuadas para facilitar una "apropiada" urbanización? Es aquí cuando pensar el territorio se convierte en un tema central. Es primordial mirar hacia las zonas rurales, marginadas y olvidadas, proyectando una manera justa de obtener el mejor provecho de este. Un campo que alimenta las ciudades, pero que continuamente es relegado y tratado como el terreno futuro para la expansión ${ }^{9}$.

Desde el POT se van dando los lineamientos para la creación de nuevos espacios públicos en zonas cercanas a la ruralidad y mejoras viales, con el fin de llevar equipamiento a las zonas hacia donde se pretende crecer. Se propicia así que estas sean más fácilmente declaradas como urbanizables, y que permitan que las inmobiliarias entren a adquirir terrenos y aumentar los precios de estos, encareciendo la vida. Por otro lado, la intención que

9 Según el POT, el suelo de expansión urbana es de 130,41 hectáreas. 
guarda revisar la estratificación es subir el estrato de algunas zonas del municipio, favoreciendo que los servicios públicos aumenten y las personas que habitan el territorio deban irse, porque ya no están en capacidad para permanecer en lugares que empiezan a resultar costosos, todo ello trae consigo el encarecimiento de la tierra y genera especulación inmobiliaria.

Todos estos acontecimientos fueron ocurriendo en la cuenca Los Gómez, cuyas dinámicas estaban relacionadas con el campo, lo agrícola y la creación de ladrillos artesanales, y que "lastimosamente" se encuentra muy cercana a la cabecera municipal. Las administraciones y los intereses privados pusieron los ojos en ella y, por medio de los planes, la fueron perfilando hasta llegar a convertirla en suelo de expansión. En este momento, los procesos de urbanización en la parte baja de la cuenca no paran, las inmobiliarias comenzaron a especular, a construir, a vender y en estructura ha cambiado significativamente. Las dinámicas sociales se modificaron, se cerraron las ladrilleras y las personas debieron conseguir sustento en otras partes; muchos optaron por vender ya que no podían afrontar los altos costos de vida. Según Calderón y Goez (2019), en esta zona se encuentran cuatro unidades cerradas, pero desde tiempo atrás, alrededor existe presencia de estas estructuras. Para citar algunos ejemplos: Ciudadela Parques de San Pablo construida por Concretec Fiduciaria de Bogotá, Laureles del Sur construida por Conaltura el 2007; entre otras, aumentaron la población de la zona en 6.447 habitantes más. Teniendo en cuenta lo anterior, es esencial equipar la zona para que estos nuevos habitantes puedan satisfacer sus necesidades, ahí se crean centros comerciales, bares, heladerías, salones de belleza, entre otros bienes y servicios que la población requiere, reproduciendo la urbanización a velocidades aceleradas.

Este proceso se repetirá una y otra vez en el territorio, ahora que el suelo de expansión ha crecido en beneficio del capital, salvaguardado por el agente que permite su proceso de reproducción, el Estado. 


\section{Referencias}

Alcaldía de Itagüí. (2000). Plan de Ordenamiento Territorial 2000-2003.

Alcaldía de Itagüí. (2004). Plan de Ordenamiento Territorial 2004-2007.

Alcaldía de Itagüí. (2008). Plan de Ordenamiento Territorial. 2008-2011.

Alcaldía de Itagüí. (2012). Plan de Ordenamiento Territorial 2012-2015.

Alcaldía de Itagüí. (2016). Plan de Ordenamiento Territorial 2016-2019.

Alcaldía de Itagüí. (2019). Presentación. http://www.itagui.gov.co/alcaldia

Arango-Escobar, G. (2008). Salvemos el espacio rural urbano colombiano. Cuarderno de Vivienda y Urbanismo, 1(2), 394-409.

Área Metropolitana. (2007). Plan integral de desarrollo metropolitano METRÓPOLI 20082020 Hacia la integración sostenible. https:/www.metropol.gov.co/planeacion/Documents/plan-metropoli-2008-2020.pdf

Calderón, D. y Goez, M. (2019) Segregación socioespacial: urbanizaciones cerradas en la parte baja de la cuenca de Los Gómez, Itagüí, Colombia. Rural y Urbano, 4(1), 79-103.

Congreso de la República de Colombia. (1994, 2 de junio). Ley 136. Por la cual se dictan normas tendientes a modernizar la organización y el funcionamiento de los municipios. Diario Oficial 41377. http://www.secretariasenado.gov.co/senado/basedoc/ley_0136_1994.html

Congreso de la República de Colombia. (2013, 29 de Abril). Ley 1625. Por la cual se deroga la Ley Orgánica 128 de 1994 y se expide el régimen para las áreas metropolitanas. Diario Oficial 48776.

Concejo Municipal de Itagüí. (2016, 24 de mayo). Acuerdo 004. Por medio del cual se adopta el plan de desarrollo del municipio de Itagüí, Antioquia para el periodo constitucional 2016-2019, denominado Itagüi avanza con equidad para todos. https://www.concejodeitagui.gov.co/acuerdos_2016/ACUERDO\%20004\%20DE\%202016.pdf

DANE. (2018). ¿Cómo vivimos? https://bit.ly/2G4iTAr

DANE. (2019). Resultados Censo Nacional de Población y Vivienda 2018 Valle de Aburrá. https://www.dane.gov.co/files/censo2018/informacion-tecnica/presentaciones-territorio/ 190822-CNPV-presentacion-Antioquia-Valle-de-Aburra.pdf

Google. (2019). [Mapa del municipio de Itagüí]. Recuperado el 20 de Febrero de 2019 de https://www.google.com/maps/place/Itag\%C3\%Bci,+Antioquia/@6.1753352,75.63143 $96,14 \mathrm{z} / \mathrm{data}=$ !3m1!4b1!4m5!3m4!1s0x8e46822e075e9cd5:0x6dd21cc8445c3d42!8m2! $3 \mathrm{~d} 6.1681832 ! 4 \mathrm{~d} 75.6190324$

Londoño, C. (1998). La apertura económica en Colombia. Pensamiento Humanista, 1(4), 39-51. 
Mesa ambiental del municipio de Itagüí. (2019). Sistema ambiental del municipio de Itagüí y su mesa ambiental del territorio. http://mesaambientalitaguil.blogspot.com/2018/08/ sistema-ambiental-del-municipio-de.html

Mészáros, I. (2001). Más allá del capital. Vadell.

Mészáros, I. (2011). Para além do capital: rumo a uma teoría da transicao. Boitempo.

Minvivienda. (2009). Vivienda de interés social usada; derecho de petición n. ${ }^{0} 4120$-E192791 de agosto 13 de 2009. https://bit.ly/3hPU0pD

Morales Soto, J. (1979). Algunos aspectos físicos de la cuenca hidrográfica de la quebrada Doña María, municipios de Itagüi y Medellín. Universidad Nacional de Colombia.

PNUD. (2019). Colombia. https://www.co.undp.org/content/colombia/es/home/countryinfo.html

Presidencia de la República de Colombia. (2017, 12 de julio). Decreto 1203. Por medio del cual se modifica parcialmente el Decreto 1077 de 2015 Único Reglamentario del Sector Vivienda, Ciudad y Territorio y se reglamenta la Ley 1796 de 2016, en lo relacionado con el estudio, trámite y expedición de las licencias urbanísticas y la función pública que desempeñan los curadores urbanos y se dictan otras disposiciones. Diario Oficial 50292. https://www.funcionpublica.gov.co/eva/gestornormativo/norma.php?i=82673

Raffestin, C. (1980). Por una geografía del poder. https://es.scribd.com/doc/140332368/LIBRO-Por-una-geografia-del-poder-RAFFESTEIN

Ribeiro, D. (2014). Estado e capital em István Mészáros. Cultura Académica.

Sposito, M. E. (1988). Capitalismo e urbanização. Contexto.

Unimedios (Agencia de Noticias UN). (2010). [Municipios del Valle de Aburrá]. Recuperado el 10 de febrero de 2020 de: http://agenciadenoticias.unal.edu.co/nc/detalle/article/ areas-metropolitanas-entre-uniones-y-problematicas/ 